\title{
Prediction of Symptomatic Embolism in Filipinos With Infective Endocarditis Using the Embolic Risk French Calculator
}

\author{
Jaime Alfonso M. Aherrera ${ }^{\mathrm{a}, \mathrm{b}}$, Maria Teresa B. Abola ${ }^{\mathrm{a}}$, Maria Margarita O. Balabagno ${ }^{\mathrm{a}}$, Lauro L. Abrahan IV ${ }^{\mathrm{a}}$, \\ Jose Donato A. Magno ${ }^{\text {a }}$ Paul Ferdinand M. Reganit ${ }^{a}$, Felix Eduardo R. Punzalan ${ }^{\mathrm{a}}$
}

\begin{abstract}
Background: Cardioembolic events are life-threatening complications of infective endocarditis (IE). The embolic risk French calculator estimates the embolic risk in IE computed on admission. Variables in this tool include age, diabetes, atrial fibrillation, prior embolism, vegetation length, and Staphylococcus aureus on culture. A computed risk of $>7 \%$ was considered high in the development of this tool. Knowledge of this risk applied in our local setting is important to guide clinicians in preventing such catastrophic complications. Among patients with IE, we aim to determine the efficacy of the embolic risk French calculator, using a computed score of $>7 \%$, in predicting major embolic events.
\end{abstract}

Methods: All adults admitted from 2013 to 2016 with definite IE were included. The risk for embolic events was computed on admission. All were monitored for the duration of admission for the occurrence of the primary outcome (any major embolic event: arterial emboli, intracranial hemorrhage, pulmonary infarcts, or aneurysms). Secondary outcomes were: 1) composite of death and embolic events; and 2) death from any cause.

Results: Eighty-seven adults with definite IE were included. Majority had a valvular heart disease and preserved ejection fraction (EF). The mitral valve was most commonly involved. Embolic events occurred in $25(29 \%)$. Multivariate analysis identified a high embolic score $>$ $7 \%$ (relative risk (RR): $15.12, \mathrm{P}<0.001$ ), vegetation area $\geq 18 \mathrm{~mm}^{2}$ (RR: 6.39, $\mathrm{P}<0.01$ ), and a prior embolism ( $R R: 5.18, \mathrm{P}=0.018$ ) to be independent predictors of embolic events. For the composite of embolic events and death, independent predictors include a high score of $>7 \%$ (RR: 13.56, $\mathrm{P}<0.001)$ and a prior embolus (RR: 13.75, $\mathrm{P}=$ $0.002)$. Independent predictors of death were a high score $>7 \%$ (RR: $6.20, \mathrm{P}=0.003)$ and $\mathrm{EF} \leq 45 \%(\mathrm{RR}: 9.91, \mathrm{P}=0.004)$.

Conclusion: Cardioembolic events are more prevalent in our study

Manuscript accepted for publication August 23, 2016

aSection of Cardiology, Department of Medicine, University of the Philippines-Philippine General Hospital, Philippines

${ }^{b}$ Corresponding Author: Jaime Alfonso M. Aherrera, Section of Cardiology, Department of Medicine, University of the Philippines-Philippine General Hospital, Philippines. Email: jimbz11@gmail.com

doi: http://dx.doi.org/10.14740/cr490w compared to previous data. The embolic risk French calculator is a useful tool to estimate and predict risk for embolic events and inhospital mortality. The risk of developing embolic events should be weighed against the risks of early preventive cardiac surgery, as to institute timely and appropriate management.

Keywords: Endocarditis; Echocardiography; Embolism; Risk calculator

\section{Introduction}

Infective endocarditis (IE) is an evolving disease. Advances in medical science have provided new insights to its pathophysiology, diagnosis, and treatment. However, even with the availability of advanced diagnostic techniques, improved antimicrobial chemotherapy, and potentially curative surgery, IE has a persistently high mortality and morbidity and has remained unchanged over the past two decades and may even be increasing [1]. One of the more catastrophic complications of IE is the occurrence of cardioembolic events to the major systems of the body, leading to adverse outcomes. They are believed to be due to fragmentation of vegetations, the prototypical lesion in IE. Risk factors for cardioembolism were identified in several reviews, but there is paucity of data in the local setting. Knowledge of these risk factors is of utmost importance to guide clinicians in prompt management to prevent such catastrophic complications.

There are two types of cardioembolic events based on duration: 1) events occurring before initiation of antibiotics, which may be prevented by early initiation of antibiotics; and 2) events that occur during or after antibiotic therapy, which may be prevented by early valve surgery. For the purposes of this study, we will focus on the predictors of embolic events that occur during antibiotic therapy. We emphasize that the evaluation of the embolic risk upon admission is critical in management of IE to institute prompt and appropriate interventions.

\section{Echocardiography and cardioembolic events}

Echocardiographic characteristics of the vegetation, the prototypical lesion in IE, have been shown in several studies to predict cardioembolic events. Of the predictors reported, the 
Table 1. Definition of Variables

\begin{tabular}{|c|c|}
\hline Variables collected & Definition/description \\
\hline Presence of minor emboli on admission & $\begin{array}{l}\text { Any minor embolic events present on admission, before the initiation of antibiotic therapy, such as } \\
\text { Janeway lesions, Roth spots, splinter hemorrhages, petechial hemorrhages, and subconjunctival } \\
\text { hemorrhage. }\end{array}$ \\
\hline Presence of major emboli on admission & $\begin{array}{l}\text { Any major embolic events present on admission, before the initiation of antibiotic therapy, further } \\
\text { defined below. }\end{array}$ \\
\hline Organism on blood culture & Organism present on at least two sets of blood cultures \\
\hline Length of vegetation & $\begin{array}{l}\text { Vegetation length measured in various planes and the maximal length was } \\
\text { recorded. For multiple vegetations, the largest length was used for analysis. } \\
\text { A vegetation length } \geq 10 \mathrm{~mm} \text { is considered to be a large vegetation. }\end{array}$ \\
\hline Area of vegetation & $\begin{array}{l}\text { Computed by the longest length multiplied by the widest width of a single vegetation. For multiple } \\
\text { vegetations, the individual computed areas were added. An area of } \geq 18 \mathrm{~mm}^{2} \text { is considered to be a } \\
\text { large vegetation. }\end{array}$ \\
\hline Significant valvular regurgitation & Defined as a "severe" regurgitant lesion (e.g., severe MR, severe TR). \\
\hline Location of vegetation & $\begin{array}{l}\text { Left-sided vegetations pertain to those located on the left side of the heart, including the MV, AV, } \\
\text { endocardium of the LA or LV, or aorta. Right-sided vegetations pertain to those located on the right } \\
\text { side, including the TV, PV, endocardium of the RA or RV, or the pulmonary artery. }\end{array}$ \\
\hline Prosthetic valve vegetation & Includes mechanical or bioprosthetic valves. \\
\hline
\end{tabular}

most frequently used were the vegetation length and/or area, mobility, and location. In a recent meta-analysis [2], the major determinant of embolic risk is the size of the vegetation, which was also included in the embolic risk French calculator. Studies in the past have varying lengths to define a "large vegetation" that is prone to embolize, but most literature would classify a vegetation length of $10-15 \mathrm{~mm}$ as "large".

\section{The embolic risk French calculator}

Hubert et al developed and validated a calculator to quantify the embolic risk of patients with endocarditis on admission [3]. The aim of their study was to develop and validate a simple calculator to quantify the embolic risk at admission of patients with IE. Eight hundred forty-seven patients with definite endocarditis were included with the primary end-point of symptomatic embolism after the initiation of antibiotic therapy up to 6 months. These events were defined as sudden clinical symptoms of cerebral ischemia or peripheral or pulmonary embolism, confirmed by corresponding imaging. Six variables were associated with embolic risks and were used to create the calculator: age, diabetes, atrial fibrillation (AF), embolism before antibiotics, vegetation length, and Staphylococcus aureus infection. The predictive accuracy of the calculator was assessed by studying the agreement between predicted embolic risk and observed embolic risk, which showed that the calibration of the model was excellent. This tool is a validated simple bedside prediction system that can be used to quantify the embolic risk at admission of patients with IE. The second part of this study validated that a high embolic risk (defined as $>7 \%$ risk) was associated with embolic events.

\section{Significance of the study}

Our study will determine the predictors of cardioembolic events. This study will also validate the embolic risk French calculator in predicting major embolic events in the local setting. Knowledge of this fatal complication is of paramount importance because early surgical intervention may be lifesaving, as long as the benefit from high-risk surgery would outweigh the risk of major embolic arterial events.

\section{Research question}

Among patients with definite IE admitted at the Philippine General Hospital (PGH) from 2013 to 2016, is a high computed embolic risk $(>7 \%)$ using the embolic risk French calculator on admission correlated with the occurrence of major cardioembolic events?

\section{Objectives}

The general objective was to determine the efficacy of a high embolic risk $(>7 \%)$ computed using the embolic risk French calculator in predicting major cardioembolic events among patients with definite infective endocarditis admitted at the University of the Philippines-Philippine General Hospital (UPPGH) from March 2013 to January 2016. 
Table 2. Definition of Major Embolic Events

\begin{tabular}{ll}
\hline Major embolic events & Definition of terms \\
\hline Arterial emboli & $\begin{array}{l}\text { Sudden interruption of blood flow to an organ system due to an embolus, presumed by the physician to } \\
\text { be from infective endocarditis. This complication is manifested by symptoms of end organ damage. For } \\
\text { example, CNS emboli - manifestations would include stroke-like symptoms/neurologic deficits, documented } \\
\text { by cranial imaging (CT or MRI). Other examples would include splenic, renal, peripheral, hepatic, retinal, } \\
\text { or mesenteric emboli. }\end{array}$ \\
Bleeding into the CNS presumed to be due to a CNS emboli, which is not attributable to any other etiology \\
such as hypertension or trauma.
\end{tabular}

The specific objectives were to describe the clinical, microbiologic, and echocardiographic profile of adult patients with IE admitted at the UP-PGH; to describe the minor and major embolic in-hospital events of adult patients admitted for IE (minor embolic events include splinter hemorrhage, Janeway lesions, Roth spots, or petechial and conjunctival hemorrhage; major embolic events include any arterial emboli, intracranial hemorrhage, pulmonary infarcts, or mycotic aneurysms); and to determine the association of a high-risk embolic risk score $>7 \%$ (as computed by the embolic risk French calculator) with outcomes within a period of 1 month after the initiation of antibiotic therapy (primary outcome: major embolic events; composite of embolic events and death; death from any cause).

\section{Methods}

\section{Study design, setting, and study population}

The study was conducted at the UP-PGH. Data were collected by chart review of admitted patients at the service and pay wards of the UP-PGH from March 2013 to January 2016. Adult patients with definite IE, based using the modified Duke's criteria, were included. The protocol was submitted to the UPMREB for ethics review and approval.

\section{Data collection}

All patients with definite IE using the modified Duke's criteria were included. The clinical, microbiologic, and echocardiographic data were gathered and recorded. The risk of the patients for embolic events was computed on admission using the French calculator, available online. Data included in the calculator include age, diabetes, AF, major embolism prior to antibiotic therapy, vegetation length on echocardiography, and Staphylococcus aureus on blood culture. After encoding these variables in the calculator, the risk for cardioembolic events was provided. In this study, a high score was defined as an embolic risk score of $>7 \%$ based on the validation study of Hubert et al. Tables 1 and 2 define the variables collected.

\section{Outcomes}

The occurrence of in-hospital major embolic events was determined (i.e. arterial emboli, intracranial hemorrhage, pulmonary infarcts, or mycotic aneurysms not attributable to any other etiology other than endocarditis). These major embolic events were diagnosed based on symptoms and/or imaging modalities. The table below defines the outcomes monitored. Other outcomes were: 1) the composite of death and embolic events; 2) death from any cause.

\section{Statistical analysis}

Descriptive analysis was done by obtaining the mean and standard deviation of quantitative variables. Proportions and frequencies were reported for qualitative variables. For quantitative variables, $t$-test of two independent samples was used to determine if there is a significant difference. For categorical variables, the Z-test was used. The logistic regression analysis was used to find correlation between the computed risk for embolic events and other echocardiographic parameters and the subsequent development of embolic events with and/or without adjustments of potentially confounding baseline variables. A P-value of $<0.25$ was considered as statistically significant. All significant variables were included in the final model of multivariate analysis.

\section{Results}

\section{Baseline characteristics}

A total of 106 patients with endocarditis were screened, but 19 were excluded due to a diagnosis of "possible IE". Eighty-seven 
Table 3. Baseline Clinical Profile Stratified by Outcome (Development of Embolus)

\begin{tabular}{|c|c|c|c|c|}
\hline Variable & $\begin{array}{l}\text { Total } \\
(n=87)(\%)\end{array}$ & $\begin{array}{l}\text { With embolus }{ }^{+} \\
(n=25)(\%)\end{array}$ & $\begin{array}{l}\text { Without embolus } \\
(n=62)(\%)\end{array}$ & P-value \\
\hline Age $($ mean $\pm \mathrm{SD})$ & $39.90(13.98)$ & $36.68(11.40)$ & $41.19(14.78)$ & 0.175 \\
\hline$>55$ years old & $15(17.24)$ & $2(8.00)$ & $13(20.97)$ & 0.147 \\
\hline$\leq 55$ years old & $72(82.76)$ & $23(92.00)$ & $49(79.00)$ & \\
\hline Male sex & $49(56.32)$ & $16(64.00)$ & $33(53.23)$ & 0.359 \\
\hline Presence of minor emboli on admission** & $44(50.57)$ & $15(60.00)$ & $29(46.77)$ & 0.264 \\
\hline Mean duration of illness in weeks & $7.21(7.75)$ & $5.00(5.41)$ & $8.10(8.38)$ & 0.092 \\
\hline$<6$ weeks (acute-subacute) & $57(65.51)$ & $19(76.00)$ & $38(61.29)$ & 0.191 \\
\hline$\geq 6$ weeks (chronic) & $30(34.48)$ & $6(24.00)$ & $24(38.71)$ & \\
\hline \multicolumn{5}{|l|}{ Organism on blood culture } \\
\hline Staphylococcus spp. & $13(14.94)$ & $7(28.00)$ & $6(9.68)$ & 0.030 \\
\hline Streptococcus viridans & $21(24.14)$ & $7(28.00)$ & $14(22.58)$ & 0.593 \\
\hline Gram negative bacteria & $11(12.64)$ & $3(12.00)$ & $8(12.90)$ & 0.909 \\
\hline Culture negative & $40(45.98)$ & $8(32.00)$ & $32(51.61)$ & 0.097 \\
\hline
\end{tabular}

${ }^{+}$Development major embolism after initiation of antibiotic therapy. ${ }^{* *}$ Minor embolic events include splinter hemorrhage, Janeway lesions or conjunctival hemorrhage.

(87) patients with a mean age of 40 years old with definite endocarditis were included. Table 3 summarizes the baseline characteristics of our patients stratified by the development of the primary outcome (major embolic events). The mean duration of illness prior to admission was 7 weeks. Majority complained of fever, worsening or development of heart failure symptoms, and edema. On admission, the most common physical examination findings were fever, signs of heart failure, and a murmur. The most common co-morbidities were diabetes mellitus, hypertension, and tuberculosis. Majority of patients had valvular and congenital heart disease as a predisposing endocardial disease (Table 4). Twenty-four (24) patients (28\%) were in AF (or had intermittent AF) documented on electrocardiogram, while the rest were in sinus rhythm. Most had negative blood cultures, but the most common organism isolated was Streptococcus virdans (24\%). Staphylococcus spp. was detected in 15\%. The most common antibiotics used were gentamycin, ceftriaxone, and vancomycin. Medical therapy for heart failure and other complications were administered as well.

\section{The embolic risk French calculator}

Components of the embolic risk French calculator include age, presence of diabetes, major emboli on admission, AF, vegetation length $\geq 10 \mathrm{~mm}$ on echocardiography, and Staphylococcus aureus on blood culture. Table 5 summarizes the variables and breakdown of the components included in the French calculator. Of note, a vegetation length $\geq 10 \mathrm{~mm}$ on echocardiography was the most frequent variable present in our database. Among those who developed embolic events, the proportion of those with major emboli prior to admission, AF, large vegetations $(\geq$

Table 4. Distribution According to Predisposing Cardiac Condition by Echocardiography

\begin{tabular}{|ll}
\hline Predisposing cardiac condition & Frequency $(\mathbf{n}=\mathbf{8 7})(\mathbf{\%})$ \\
\hline Valvular heart disease (rheumatic and non-rheumatic) & $59(68 \%)$ \\
Mitral stenosis & 25 \\
Mitral valve prolapse & 14 \\
Aortic stenosis & 9 \\
Congenital heart disease (CHD) & $15(17 \%)$ \\
Ventricular septal defect & 8 \\
Patent ductus arteriosus & 3 \\
Repaired CHD & 2 \\
Pulmonic stenosis & 1 \\
Prosthetic valve endocarditis & $5(7 \%)$ \\
Unknown or no underlying predisposition & $8(11 \%)$ \\
\hline
\end{tabular}


Table 5. Components of the French Risk Calculator

\begin{tabular}{|c|c|c|c|c|}
\hline Variable & $\begin{array}{l}\text { Total } \\
(n=87)(\%)\end{array}$ & $\begin{array}{l}\text { With embolus }{ }^{+} \\
(n=25)(\%)\end{array}$ & $\begin{array}{l}\text { Without embolus } \\
(\mathrm{n}=62)(\%)\end{array}$ & P-value \\
\hline Mean age (SD) & $39.86(13.95)$ & $36.72(11.39)$ & $41.13(14.75)$ & 0.183 \\
\hline Presence of diabetes mellitus & $18(20.69)$ & $6(24.00)$ & $12(19.35)$ & 0.628 \\
\hline Major emboli prior to admission & $18(20.69)$ & $11(44.00)$ & $7(11.29)$ & $0.001 *$ \\
\hline Atrial fibrillation & $23(26.44)$ & $12(48.00)$ & $11(17.74)$ & $0.004^{*}$ \\
\hline \multicolumn{5}{|l|}{ Length of vegetation on 2D echocardiogram } \\
\hline$<10 \mathrm{~mm}$ & $51(58.62)$ & $7(28.00)$ & $44(70.97)$ & $<0.001 *$ \\
\hline$\geq 10 \mathrm{~mm}$ & $35(40.23)$ & $18(72.00)$ & $17(27.42)$ & $<0.001 *$ \\
\hline Staphylococcus aureus on blood culture & $8(9.20)$ & $6(24.00)$ & $2(3.23)$ & $0.002 *$ \\
\hline Mean score on French calculator (SD) & $5.13(4.33)$ & $8.68(5.75)$ & $3.69(2.48)$ & $<0.001 *$ \\
\hline
\end{tabular}

${ }^{+}$Major embolic events included any arterial emboli, intracranial hemorrhage, pulmonary infarcts, or mycotic aneurysms. ${ }^{*} \mathrm{P}$ value $<$ 0.05 .

$10 \mathrm{~mm}$ ), and Staphylococcus aureus was more frequent. The mean score derived from the French calculator was significantly higher among those who developed embolic events $(8.68 \%$ vs. $3.69 \%, \mathrm{P}<0.001)$.

\section{Echocardiographic profile of patients included}

The systolic function was generally preserved in our sample, with a mean ejection fraction (EF) of $61 \%$. Only a small percentage $(13 \%)$ had a depressed EF of $\leq 45 \%$. The most common valve involvement (of vegetations) was the mitral (64\%), followed by the aortic valve (24\%). Involvement of the tricuspid and/or pulmonic valve was uncommon (9\%). Severe regurgitant lesions were seen in $45 \%$. As mentioned, majority had an underlying valvular heart disease, most common of which were mitral valve prolapse, mitral regurgitation, and mitral stenosis. Among those with congenital heart diseases, common defects included ventricular septal defect, valvular lesions, and a patent ductus arteriosus. Prosthetic valve endocarditis was diagnosed in only three patients $(3 \%)$. In 19 patients, a transesophageal echocardiogram was needed to confirm the diagnosis of endocarditis. Majority had at least one vegetation documented (74\%), while some had multiple vegetations $(21 \%)$. Majority of the vegetations were less than 10 $\mathrm{mm}$ in longest length (57\%), while only $16 \%$ had a vegetation $>15 \mathrm{~mm}$ in length. The area of the vegetation was computed by multiplying the longest length and the widest width. Our sample had a mean vegetation area of $70.4 \mathrm{~mm}^{2}$ and majority $\left(57 \%\right.$ ) had an area of $\geq 18 \mathrm{~mm}^{2}$ (value of significance in some studies reviewed). Table 6 summarizes the echocardiographic profile of our patients, stratified by the development of the primary outcome (major embolic event).

\section{Description of the minor and major embolic events en- countered in the study}

Minor and major emboli were present on admission in $51 \%$ and $30 \%$, respectively. Minor emboli included subconjunctival hemorrhage (37\%), Janeway lesions (22\%), Roth spots (14\%), splinter hemorrhages $(8 \%)$, and non-specific rashes or petechiae were seen in 34\%. Seventeen (17) patients had major embolic events prior to therapy (i.e., present on admission). Twenty-five (25) patients had new embolic events during the course of therapy (primary outcome). Common sites of major embolism were the CNS, pulmonary, and renal vasculature. Figure 1 summarizes the distribution of these embolic events. Of note, six patients had embolic events in more than one organ system.

\section{Primary endpoint: predictors of major embolic events during admission}

Major embolic events after the initiation of antibiotic therapy developed in 25 patients (29\%). Majority occurred during the first week of antibiotic therapy (47\%). Among those who eventually developed an embolus at 1 month (primary outcome), the proportions of those who had a previous major embolus on admission and AF on electrocardiogram were higher, compared to those who did not have an embolism at 1 month. Those who had a major embolus during the course also had larger vegetations (length $\geq 10 \mathrm{~mm}$ and/or area $\geq$ $18 \mathrm{~mm}^{2}$ ) on 2D echocardiogram, compared to those who did not develop embolic events. Of note, the mean embolic risk French score computed in the calculator was higher among those who developed a cardioembolism compared to those who did not develop a cardioembolism $(8.7 \%$ vs. $3.7 \%$, respectively). Other than the vegetation length and area, the other echocardiographic parameters did not statistically differ among the two groups: those who had embolic events had more participants with a vegetation length $\geq 10 \mathrm{~mm}$ and area $\geq 18 \mathrm{~mm}^{2}$.

All patients were classified according to the value of their embolic risk predicted by the calculator at 1 month after initiation of antibiotic therapy (high versus low risk). Two groups were formed: greater than $7 \%$ vs. $\leq 7 \%$. This was the 
Table 6. Echocardiographic Profile Stratified by Outcome (Development of Embolus)

\begin{tabular}{|c|c|c|c|c|}
\hline Variable & $\begin{array}{l}\text { Total } \\
(n=87)(\%)\end{array}$ & $\begin{array}{l}\text { With embolus }^{+} \\
(n=25)(\%)\end{array}$ & $\begin{array}{l}\text { Without embolus } \\
(n=62)(\%)\end{array}$ & P-value \\
\hline \multicolumn{5}{|l|}{ Overall cardiac structure and function } \\
\hline Mean ejection fraction (SD) & $60.92(11.57)$ & $60.32(12.70)$ & $61.16(11.18)$ & 0.761 \\
\hline Ejection Fraction $<45 \%$ & $11(12.64)$ & $5(20.00)$ & $6(9.68)$ & 0.190 \\
\hline Mean LAVI $\left(\mathrm{mL} / \mathrm{m}^{2}\right)^{++}$ & $44.56(29.84)$ & $41.40(25.76)$ & $45.84(31.44)$ & 0.533 \\
\hline $\mathrm{LAVI}>34 \mathrm{~mL} / \mathrm{m}^{2}$ & $54(62.07)$ & $17(68.00)$ & $37(59.68)$ & 0.469 \\
\hline Significant valvular regurgitation & $39(44.83)$ & $12(48.00)$ & $27(43.55)$ & 0.706 \\
\hline \multicolumn{5}{|l|}{ Number of vegetations } \\
\hline 0 vegetation & $5(5.75)$ & $2(8.00)$ & $3(4.84)$ & 0.566 \\
\hline 1 vegetation & $64(73.56)$ & $16(64.00)$ & $48(77.42)$ & 0.199 \\
\hline$>1$ vegetation & $18(20.69)$ & $7(28.00)$ & $11(17.74)$ & 0.285 \\
\hline \multicolumn{5}{|l|}{ Size and characteristics of the vegetation } \\
\hline Vegetation length (mean $\pm \mathrm{SD})$ & $8.45(6.11)$ & $10.60(6.49)$ & $7.58(5.78)$ & $0.036^{*}$ \\
\hline Vegetation length $\geq 10 \mathrm{~mm}$ & $35(40.23)$ & $16(64.00)$ & $19(30.65)$ & $0.004 *$ \\
\hline Vegetation length $>15 \mathrm{~mm}$ & $14(16.09)$ & $6(24.00)$ & $8(12.90)$ & 0.202 \\
\hline Vegetation area $(\text { mean } \pm \mathrm{SD})^{+++}$ & $70.40(85.22)$ & $112.72(100.97)$ & $53.33(72.09)$ & $0.003 *$ \\
\hline Vegetation area $\geq 18 \mathrm{~mm}^{2}$ & $50(57.47)$ & $21(84.00)$ & $29(46.77)$ & $0.001^{*}$ \\
\hline Mobile vegetation & $61(70.11)$ & $21(84.00)$ & $40(64.52)$ & 0.072 \\
\hline Presence of abscess & $6(6.90)$ & $2(8.00)$ & $4(6.45)$ & 0.796 \\
\hline \multicolumn{5}{|l|}{ Location of vegetation } \\
\hline Left-sided vegetation & $79(90.80)$ & $21(84.00)$ & $58(93.55)$ & 0.163 \\
\hline Right-sided vegetation & $10(11.49)$ & $5(20.00)$ & $5(8.06)$ & 0.114 \\
\hline \multicolumn{5}{|l|}{ Valvular involvement } \\
\hline Mitral location & $56(64.37)$ & $17(68.00)$ & $39(62.90)$ & 0.653 \\
\hline Aortic location & $21(24.14)$ & $7(28.00)$ & $14(22.58)$ & 0.593 \\
\hline Tricuspid/pulmonic location & $8(9.20)$ & $5(20.00)$ & $3(4.84)$ & $0.027 *$ \\
\hline Multiple valves & $19(21.84)$ & $8(32.00)$ & $11(17.74)$ & 0.145 \\
\hline Prosthetic valves & $3(3.45)$ & $1(4.00)$ & $2(3.23)$ & 0.858 \\
\hline No valves (endocardium) & $10(11.49)$ & $3(12.00)$ & $7(11.29)$ & 0.925 \\
\hline
\end{tabular}

${ }^{+}$Development major embolism after initiation of antibiotic therapy. ${ }^{++}$Left atrial volume index. ${ }^{+++}$Vegetation area was computed by multiplying the longest length with the widest width. ${ }^{*} \mathrm{P}$-value $<0.05$.

cut-off value validated by Hubert et al. Univariate analysis (Table 7) was done and identified significant variables associated with the occurrence of embolic events: high computed risk for embolic events $(>7 \%)$, embolism prior to admission, AF, vegetation size on echocardiography (length $\geq 10 \mathrm{~mm}$ and/or area $\geq 18 \mathrm{~mm}^{2}$ ), and Staphylococcus aureus on blood culture. Of these, multivariate analysis (Table 8) identified a high computed embolic risk French score $(>7 \%)$, a vegetation area of $\geq 18 \mathrm{~mm}^{2}$, and the presence of an embolism prior to initiation of medical therapy to be significant and independent predictors of embolic events. The risk of having a major embolic event among those with a high computed embolic risk using the French calculator $(>7 \%)$ is 15.1 times higher than those with a low computed score (relative risk (RR): 14.1, $\mathrm{P}<0.001)$.

\section{Secondary endpoints: composite of embolic events and death during admission}

Secondary endpoints tested include a composite of death and embolic events and death from any cause. Thirty-seven patients $(43 \%)$ had the outcome of composite of death and embolic events. There were 25 mortalities $(29 \%)$ in our sample population. The most common cause of death in our data was presumed to be sequelae of cardioembolic events such as brain 


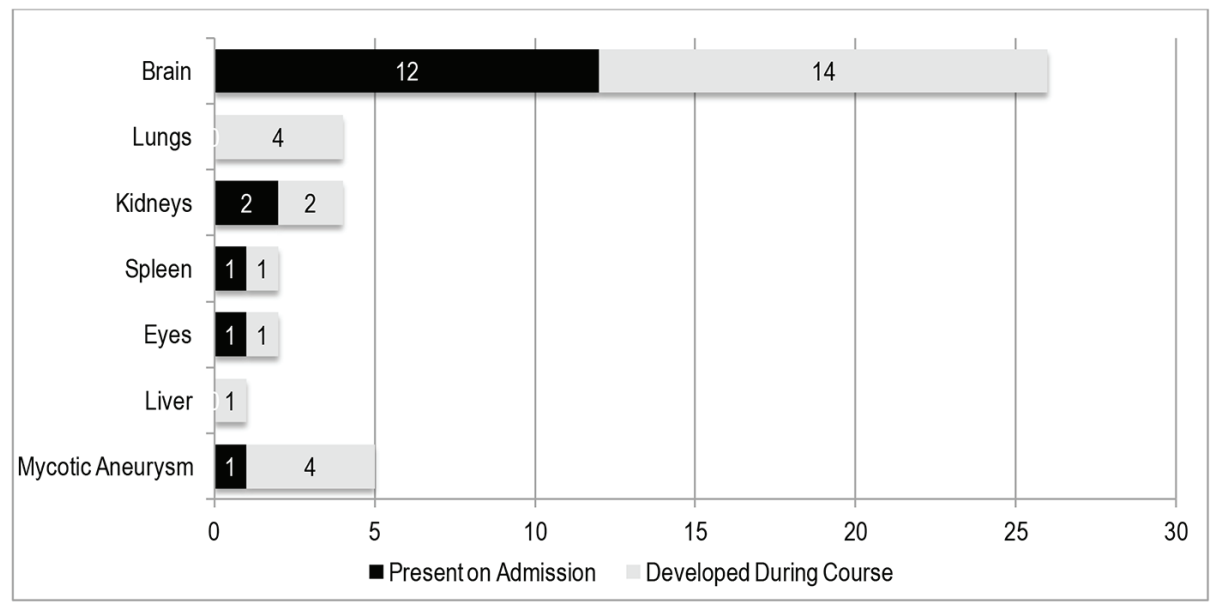

Figure 1. Distribution of cardioembolic events. Embolic events were present on admission (prior to initiation of antibiotic therapy) in 17 patients. The primary outcome (i.e. embolic events during/after initiation of antibiotic therapy) occurred in 25 patients.

herniation from CNS emboli or intracranial hemorrhage, respiratory failure from pulmonary emboli, or sepsis from solid organ abscesses. Other causes of death were heart failure, fatal arrhythmias, and sepsis. Figure 2 illustrates the causes of death in our patients.

For the composite of major embolic events and death, significant independent predictors on multivariate analysis include a high computed score $>7 \%$ (RR: 13.56, CI: $3.20-57.5$, $\mathrm{P}<0.001$ ) and a major embolus prior to admission (RR: 13.75, $\mathrm{CI}: 2.55$ - 74.11, $\mathrm{P}=0.002)$. Significant independent predictors of death from any cause on multivariate analysis are a high computed score $>7 \%$ (RR: 6.20, CI: $1.89-20.32, \mathrm{P}=0.003$ ) and a $2 \mathrm{D}$ echocardiographically derived $\mathrm{EF} \leq 45 \%$ (RR: 9.91 , CI: 2.11 - 46.63, $\mathrm{P}=0.004$ ). Table 9 summarizes the significant predictors on multivariate analysis for primary (discussed above) and secondary outcomes.

\section{Discussion}

\section{Embolic events in endocarditis}

Embolic events in endocarditis are frequent and life-threatening complications, believed to be caused by fragmentation of vegetations [4]. Major embolic events include arterial emboli, intracranial hemorrhage, pulmonary infarcts, or mycotic aneurysms; while minor embolic events include splinter hemorrhage, Janeway lesions or conjunctival hemorrhage. In a local retrospective study by Javier et al in 2009 of 91 patients with definite endocarditis, vascular manifestations included Roth spots (1\%), splinter hemorrhages (1\%), and Janeway lesions (1\%) [5]. Another retrospective study by Pasaporte and Pena from 1979 to 2001 included 239 patients.

Table 7. Univariate Analysis to Predict Development of Embolic Events during Admission

\begin{tabular}{llll}
\hline Variable & Relative risk & $\mathbf{9 5 \%}$ CI & P-value \\
\hline High risk for embolic events $(>7 \%)^{*}$ & 20.27 & $5.95-69.05$ & $<0.001^{*}$ \\
Age $>55$ & 0.33 & $0.07-1.57$ & 0.163 \\
Diabetes mellitus & 1.04 & $0.32-3.34$ & 0.945 \\
Minor embolism prior to therapy & 1.71 & $0.66-4.38$ & 0.266 \\
Major embolism prior to therapy & 8.62 & $2.73-27.23$ & $<0.001^{*}$ \\
Atrial fibrillation & 3.85 & $1.41-10.52$ & $0.009^{*}$ \\
Ejection fraction $<45 \%$ & 2.33 & $0.64-8.49$ & 0.199 \\
Left-sided vegetation & 0.36 & $0.08-1.58$ & 0.176 \\
More than one vegetation & 1.80 & $0.61-5.36$ & 0.289 \\
Vegetation length $\geq 10 \mathrm{~mm}$ & 4.02 & $1.51-10.71$ & $0.005^{*}$ \\
Vegetation length $\geq 15 \mathrm{~mm}$ & 2.13 & $0.65-6.94$ & 0.209 \\
Area of vegetation $\geq 18 \mathrm{~mm}{ }^{2}$ & 5.97 & $1.84-19.44$ & $0.003^{*}$ \\
Staphylococcus aureus on culture & 3.63 & $1.08-12.21$ & $0.037^{*}$ \\
\hline
\end{tabular}

+Included in the final model of multivariate analysis. *High risk embolic events using the embolic risk French calculator were set at $>7 \%$ risk at 1 month (see references). 
Table 8. Multivariate Analysis to Predict Embolic Events

\begin{tabular}{llll} 
Variable & Relative risk & $\mathbf{9 5 \%}$ CI & P-value \\
\hline High risk score for embolic events $(>7 \%)^{*}$ & 15.12 & $4.19-54.54$ & $<0.001^{+}$ \\
Vegetation area $\geq 18 \mathrm{~mm}^{2}$ & 6.39 & $1.55-26.31$ & $0.010^{+}$ \\
Major embolism prior to therapy & 5.18 & $1.32-20.35$ & $0.018^{+}$ \\
\hline
\end{tabular}

+Independent predictors of embolic events with $\mathrm{P}$ value $<0.05$. *High risk embolic events using the embolic risk French calculator were set at $>7 \%$ risk at 1 month.

Vascular manifestations included splinter hemorrhages (4\%), Janeway lesions (2\%), Roth spots (1\%), and embolic phenomenon $(6 \%)$ [6].

Our data, collected prospectively, showed a higher prevalence of minor and major vascular events. Consistent with available literature, the most common site of major embolic events in our study was still the CNS, manifesting as neurologic deficits, headache, dizziness, seizures, or loss of consciousness. Pulmonary embolism manifested as hemoptysis, sudden respiratory failure, and/or hypoxemia. Renal and splenic emboli manifested as abdominal pain or tenderness, document by ultrasound in most cases (autopsy in one case). Embolism to the eye(s) was seen in two patients, which manifested as endophthalmitis. Aneurysms occurred in three patients (mycotic aneurysm in two, and celiac aneurysm in another). Of note, the occurrence of embolic events was associated with in-hospital mortality [7].

\section{Echocardiographic predictors of major embolic events in endocarditis}

Advances in echocardiographic techniques have brought about a better understanding of the correlation between the echocardiographic characteristics of the vegetation and incidence of complications. Predictors of the occurrence of embolic events and its prognostic influence were also reviewed by Fabri et al [8] among 629 patients with left-sided endocarditis. Among this population, 133 patients had embolic events. Embolic events were higher in $S$. aureus IE, mitral or aortic prosthetic valve endocarditis, and vegetations on echocardiography. They were lower in those with a longer duration of symptoms. The presence of embolic events also doubled the risk of mortality in this population. Di Salvo et al [9], in their review of 178 patients with IE (66 of whom had embolic events), con- cluded that the only predictors of embolism were vegetation length $(>15 \mathrm{~mm})$. There was no difference between patients with and without embolism in terms of age, gender and left valve involved. Contrary to traditional knowledge, another review by De Castro et al [10] concluded that there were no differences in any of the echocardiographic characteristics of embolic events.

The vegetation size seems to be the most studied and most consistent echocardiographic parameter with regard to predictors of cardioembolic events. In our review of literature, there are two methods to describe vegetation size: 1) vegetation length $(\mathrm{mm})$, and 2$)$ vegetation area $\left(\mathrm{mm}^{2}\right)$. A large vegetation would usually pertain to a vegetation that is more than $10-15 \mathrm{~mm}$ in length, which is a predictor of cardioembolic events in IE. In a systematic review of Okonta and Adamu [11] involving 16 papers, a vegetation length of $\geq 10 \mathrm{~mm}$ is an indication for surgery. A study by Kjaergaard et al [12] found that vegetation area performed better than the vegetation length in predicting adverse. Since it is not routine for cardiologists to measure the size of vegetation using the area $\left(\mathrm{mm}^{2}\right)$, this finding may encourage its utility in IE patients. Our study has demonstrated that a vegetation length $>10 \mathrm{~mm}$ (as a component of the French embolic risk score) and a vegetation area $\geq 18 \mathrm{~mm}^{2}$ are two useful parameters to identify those who are at risk for embolic events during antibiotic therapy.

\section{The embolic risk French calculator}

The vegetation length and the presence of a previous embolism are the only two parameters included in the present international guidelines to assess embolic risk and indicate preventive valve surgery $[13,14]$. As mentioned in the paper of Hubert et al, these recommendations do not provide a precise quantifica-

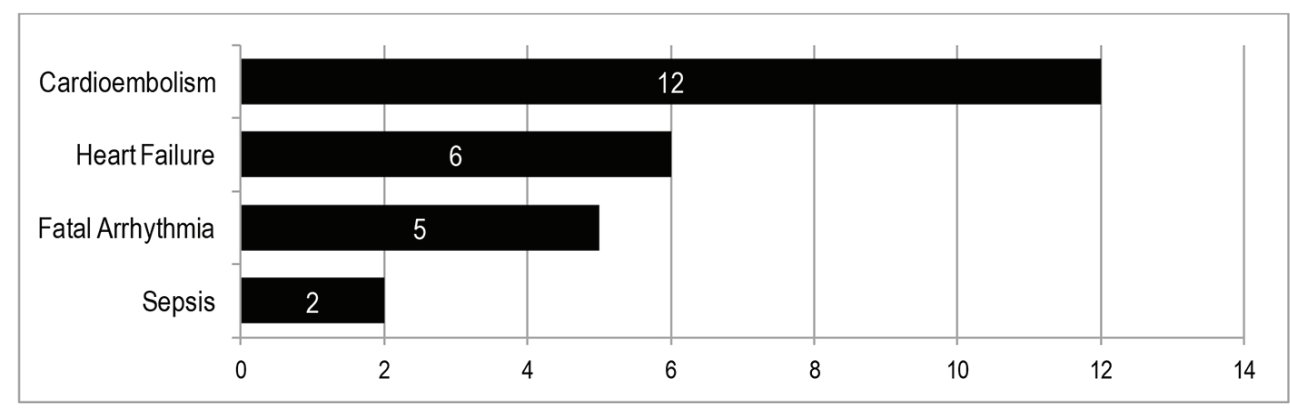

Figure 2. Causes of death. Mortality occurred in 25 patients (33\%) prior to the 28-day observation period. 
Table 9. Multiple Logistic Regression to Predict Primary and Secondary Outcomes

\begin{tabular}{|c|c|c|c|c|c|c|}
\hline \multirow{2}{*}{ Variable } & \multicolumn{2}{|c|}{ Embolic event (primary endpoint) } & \multicolumn{2}{|c|}{ Composite of death and embolic events } & \multicolumn{2}{|c|}{ Death } \\
\hline & RR & P-value & $\mathbf{R R}$ & P-value & $\mathbf{R R}$ & P-value \\
\hline High risk score $(>7 \%) *$ & 15.12 & $<0.001$ & 13.56 & $<0.001$ & 6.2 & 0.003 \\
\hline Emboli prior to admission & 5.18 & 0.018 & 13.75 & 0.002 & NS & \\
\hline Ejection fraction $\leq 45 \%$ & NS & & NS & & 9.91 & 0.004 \\
\hline
\end{tabular}

*High risk score pertains to a high embolic risk score at one month, calculated using the French calculator. RR: relative risk; NS: not significant.

tion of embolic risk and do not take into account other potentially important predictors. The embolic risk French calculator, in addition to vegetation length and previous embolism, also takes into account variables that were related to patient characteristics (age, diabetes, and AF). This calculator focused on predictors on admission, as these were the most important factors in rapid therapeutic decisions to avoid embolic events. Our data validated the use of the embolic risk French calculator to predict embolic events. Likewise, since embolic events were related to mortality in IE, a high-risk score on the calculator was also a predictor of death.

\section{Death and endocarditis}

Among the 87 patients, 25 (29\%) died before completing the 28-day observation period. Majority of the deaths were presumed to be sequalae of cardioembolic events, therefore, embolic events should also be regarded as an indicator of increased risk for mortality emphasizing the prognostic significance of this complication. A previous paper by Aherrera et al [8] for publication in the Philippine Journal of Cardiology demonstrated that the occurrence of embolic events is also a predictor of death (RR: 4.5, CI: 1.41-14.3, P = 0.011). Our study has shown that a high embolic risk score using the French calculator and a low EF during IE are predictors of death from any cause.

\section{Limitations of the study}

It is important to note that the outcome of cardioembolism was investigated only when presented with symptoms, therefore, asymptomatic emboli may have been undetected. Imaging modalities were not done in all patients; rather, they were guided by the presence of symptoms. Therefore, microemboli causing minimal or no symptoms may have been undetected. In addition, 25 patients (29\%) died before the end of the 28-day observation period; however, 12 of the 25 patients (48\%) died as a sequala of cardioembolism. Therefore, the outcome of cardioembolism may be underestimated due to the 13 deaths that did not reach the 28 th day of observation.

Given these limitations, we recommend that a larger, multicenter study be done that would have more precise outcome assessors, such as standardized imaging (i.e., cranial CT or MRI, abdominal CT, etc.) to be done to all patients at the end of the observation period.

\section{Conclusion}

Major and minor embolic manifestations in endocarditis were prevalent in our study. The most common site of major embolism was the CNS. Emboli occur relatively early in the course of IE, even during antibiotic therapy. The French risk calculator and echocardiography are useful tools to estimate the risk for embolic events and death among patients with definite endocarditis in our setting. Our study has demonstrated that echocardiographic parameters such as a vegetation length $\geq 10$ $\mathrm{mm}$ (as a component of the French embolic risk score) and a vegetation area $\geq 18 \mathrm{~mm}^{2}$ are two useful parameters to identify those who are at risk for embolic events during antibiotic therapy. The risk for embolism in IE should be quantified at admission using the specified predictors to make rapid therapeutic decisions. This risk should be weighed against the risks of early cardiac surgery during the active phase of endocarditis, as to institute timely and appropriate management.

\section{References}

1. Prendergast BD. The changing face of infective endocarditis. Heart. 2006;92(7):879-885.

2. Salehian O, Chan KL. Systemic embolism in endocarditis: incidence, risk factors, clinical significance, and treatment strategies. In: Chan KL, Embil JM. Endocarditis. Diagnosis and Management. London: Springer-Verlag; 2006:229-240.

3. Hubert S, Thuny F, Resseguier N, Giorgi R, Tribouilloy C, Le Dolley Y, Casalta JP, et al. Prediction of symptomatic embolism in infective endocarditis: construction and validation of a risk calculator in a multicenter cohort. J Am Coll Cardiol. 2013;62(15):1384-1392.

4. Thuny F, Habib G, Le Dolley Y, Canault M, Casalta JP, Verdier M, Avierinos JF, et al. Circulating matrix metalloproteinases in infective endocarditis: a possible marker of the embolic risk. PLoS One. 2011;6(4):e18830.

5. Javier D, et al. The changing face of infective endocarditis in the Filipino: A six year review (2004-2009) at the Philippine General Hospital. Unpublished.

6. Pasaporte BB, Pena AC. Association of infective endocarditis with age: a 21 years experience. Philippine Journal of Microbiology. 33:1.

7. Aherrera J, Balabagno M, et al. Risk of death and adverse outcomes in adult Filipinos admitted for infective endocarditis: A prospective cohort. Philippine Journal of Car- 
diology. 42:1.

8. Fabri J, Jr., Issa VS, Pomerantzeff PM, Grinberg M, Barretto AC, Mansur AJ. Time-related distribution, risk factors and prognostic influence of embolism in patients with left-sided infective endocarditis. Int $\mathrm{J}$ Cardiol. 2006;110(3):334-339.

9. Di Salvo G, Habib G, Pergola V, Avierinos JF, Philip E, Casalta JP, Vailloud JM, et al. Echocardiography predicts embolic events in infective endocarditis. J Am Coll Cardiol. 2001;37(4):1069-1076.

10. De Castro S, Cartoni D, et al. Transthoracic and transesophageal echocardiographic findings of vegetative lesions are not predictive of subsequent embolic events in patients with active infective endocarditis. JASE. 1997;9:406.

11. Okonta KE, Adamu YB. What size of vegetation is an indication for surgery in endocarditis? Interact Cardiovasc Thorac Surg. 2012;15(6):1052-1056.

12. Kjaergaard J, Rasmussen R, Bruun N, et al. Vegetation length or area: which is the better predictor of outcome in infective endocarditis? Unpublished data.
13. Baddour LM, Wilson WR, Bayer AS, Fowler VG, Jr., Bolger AF, Levison ME, Ferrieri P, et al. Infective endocarditis: diagnosis, antimicrobial therapy, and management of complications: a statement for healthcare professionals from the Committee on Rheumatic Fever, Endocarditis, and Kawasaki Disease, Council on Cardiovascular Disease in the Young, and the Councils on Clinical Cardiology, Stroke, and Cardiovascular Surgery and Anesthesia, American Heart Association: endorsed by the Infectious Diseases Society of America. Circulation. 2005;111(23):e394-434.

14. Habib G, Hoen B, Tornos P, Thuny F, Prendergast B, Vilacosta I, Moreillon P, et al. Guidelines on the prevention, diagnosis, and treatment of infective endocarditis (new version 2009): the Task Force on the Prevention, Diagnosis, and Treatment of Infective Endocarditis of the European Society of Cardiology (ESC). Endorsed by the European Society of Clinical Microbiology and Infectious Diseases (ESCMID) and the International Society of Chemotherapy (ISC) for Infection and Cancer. Eur Heart J. 2009;30(19):2369-2413. 